\title{
Acute severe headaches in pregnancy are a 'red flag': A review based on case reports and key messages for healthcare practioneers
}

\author{
J Moodley, ${ }^{1}$ MB ChB, FCOG (SA), FRCOG, MD; N Mayat, ${ }^{2}$ MB ChB, FCOG (SA); N Moran, ${ }^{3}$ MB ChB, FCOG (SA) \\ ${ }^{1}$ Women's Health and HIV Research Group, Department of Obstetrics and Gynaecology, School of Clinical Medicine, \\ College of Health Sciences, University of KwaZulu-Natal, Durban, South Africa \\ ${ }^{2}$ Department of Obstetrics and Gynaecology, Queen Nandi Hospital, Empangeni, South Africa \\ ${ }^{3}$ KwaZulu-Natal Provincial Department of Health, Durban, South Africa
}

Corresponding author: J Moodley (jmog@ukzn.ac.za)

Headaches are common in pregnancy and the immediate postpartum period. Fortunately $>90 \%$ of these headaches are primary and benign Generally, the most common primary headache disorders have a peak incidence at a younger age and affect women disproportionately, especially in the reproductive phase of life. The higher incidence in females may reflect the relationship between headache and sex hormones, particularly oestrogen. In pregnancy, most headaches in the first trimester are primary, while the incidence of secondary headaches increases in the third trimester and the immediate postpartum period. This probably reflects when pre-eclampsia and changes in the coagulation system are the greatest. Despite this, 50 - 70\% of headaches in late pregnancy have a primary benign cause. Common causes of headaches in pregnancy and the postpartum period are: migraine (vascular), tension headache (brain tumour), cluster headache (severe pre-eclampsia/eclampsia) and cough (postdural puncture, reversible cerebral vasoconstriction syndrome, idiopathic intracranial hypertension, cerebral venous thrombosis). This article presents two cases of maternal deaths arising from lack of awareness that acute severe headaches in pregnancy may have serious underlying pathology, and that persistent headache should be regarded as a 'red flag', as suggested by neurologists.

S Afr Med J 2018;108(10):807-808. DOI:10.7196/SAMJ.2018.v108i10.13060

\section{Case reports}

A 20-year-old primigravida presented at a district hospital with a complaint of persistent headache and dizziness that did not respond to non-opioid analgesics. On examination, she was 14 weeks' pregnant, apyrexial, with a pulse rate of $74 \mathrm{bpm}$ and a blood pressure of $140 / 60 \mathrm{mmHg}$, and was HIV-negative. The patient was also irritable. Despite this, a thorough neurological and ophthalmological examination was not done, and non-opioid analgesics and antiemetics were prescribed, presumably because she was thought to have symptoms of early pregnancy. The patient's headache and irritability persisted for the next 48 hours, when a lumbar puncture was done without seeking the opinion of a neurologist.

The cerebrospinal fluid examination did not reveal any abnormality and the patient's neurological condition deteriorated rapidly during the next 24 hours, without any further investigation. An autopsy revealed a cerebral tumour and cerebral oedema.

The second patient was also managed at a district hospital in a rural area. This patient (parity 1, gravida 2) booked for antenatal care at 12 weeks' gestation. Two previous antenatal visits were uneventful. At 32 weeks' gestational age she complained of severe headache and vomiting for a week.

The patient was admitted and treated for gastroenteritis and possibly hyperemesis gravidarum; an anti-emetic (metoclopramide (Maxolon)) was prescribed. During the next 3 weeks her headaches persisted and at 35 weeks she developed nystagmus, but without neurological signs. The patient was HIV-negative and liver function tests, including haemoglobin and platelets, were normal. A gynaecological consultation led to induction of labour and referral to a tertiary hospital for a head scan, which revealed metastatic tumours in the posterior cerebrum and a hydrocephalus. The latter was drained, but the patient died 2 days later without the site of the main tumour being identified.

\section{Discussion}

These two patients illustrate that inexperienced doctors working in district hospitals in SA are not aware that severe persistent headache in pregnancy is a 'red flag' and warrants a neurological opinion. Most authors regard persistent severe headache as those continuing for $>72$ hours. ${ }^{[1,2]}$ Moreover, the second case illustrates that severe headache occurring for the first time in the third trimester should have alerted the doctors to a secondary cause. ${ }^{[3]}$ Severe headache in the third trimester, especially when associated with other signs such as vomiting and visual disturbances (e.g. scotomata, photopsia, diplopia, blurry vision) as in the second patient presented in this report, may be associated with pre-eclampsia. ${ }^{[4]}$

Headache is considered a promontory symptom for eclampsia, although it is only present in $56 \%$ of patients with eclampsia. ${ }^{[5]}$ However, the incidence of headache is far greater in patients with pre-eclampsia; a case-control study showed an odds ratio of 4.95 (95\% confidence interval 2.47 - 9.92) of headache being more frequent in pre-eclampsia. ${ }^{[6]}$ No single type of headache, such as throbbing pain, generalised pressure, knife-like sensations or piercing, has been reported, but none of the above is typical of pre-eclampsia. ${ }^{[6]}$

The patients presented did not have hypertension and/or proteinuria. Furthermore, the platelet counts and liver function tests were normal. Atypical presentations of headache, persistent 
symptomatology or focal neurological signs warrant consideration of other secondary causes of headache, such as tumours non-related to pre-eclampsia or intracranial pathology related to pre-eclampsia. ${ }^{[7,8]}$

There is a reluctance to perform neuroimaging in pregnancy, even though it is often necessary. The major concern with radiological investigations is fetal safety. Magnetic resonance imaging as the preferred modality during pregnancy has been proven. Gadolinium is not used because it crosses the placental barrier. ${ }^{\left[{ }^{-11]}\right.}$ However, in a neurological emergency, a computed axial tomography image is often the most appropriate modality to obtain timely information. The amount of radiation exposure from a non-contrast maternal head scan is only $200 \mathrm{mrad}$ - a dose much less than that shown to cause fetal death or anomalies. ${ }^{[10,11]}$

The two patients described above also illustrate the need to obtain a detailed history of the complaint of headache in pregnancy. New onset of headache in the third trimester of pregnancy or atypical headache at any stage of pregnancy, should alert a health professional to the likelihood of a secondary cause. Melhado et al., ${ }^{[12]}$ in a study of 1029 pregnant women, found a subgroup of 76 women with newonset or a new type of headache in pregnancy, of whom $52.5 \%$ had a secondary headache. The most common causes were migraine (34.2\%) and headache due to hypertension (32.9\%).

Various retrospective and prospective studies have shown the frequency of migraine attacks to decrease by the end of the second trimester. However, because the headache and related symptoms of pre-eclampsia and migraine overlap, close follow-up with monitoring for signs of pre-eclampsia is necessary. It has also been shown that women with migraine are more likely to develop pre-eclampsia. ${ }^{[1]}$

In the second patient, metoclopramide was used as an antiemetic agent. This drug has generally been regarded as safe to use in pregnancy. However, clinicians should be aware that the use of this antidopaminergic agent can cause maternal extrapyramidal symptoms, such as acute dystonic reactions and dyskinesia, which may have occurred in this patient. ${ }^{[12]}$ A careful medication history must be taken. This is of particular importance in SA owing to the high incidence of HIV infection in pregnancy and the potential side-effects, such as headache from antiretroviral agents and calcium channel antihypertensive agents, e.g. nifedipine.

In general, headaches in pregnancy are common - most are primary and benign. However, secondary causes of headache have dire consequences. The key messages warranting attention are shown below. Urgent neurological opinions are difficult to obtain in rural areas. Therefore, health authorities must consider setting up guidelines for neurological expert opinion.

\section{Key messages}

- Headaches in pregnancy are common, usually occur in the first trimester and are related to a primary headache disorder.
- During the last trimester and the postpartum period, there is an increased risk of secondary headaches.

- Be aware of new-onset, atypical headaches and persistent headaches not responding to non-opioid analgesics.

- Be aware of headaches associated with hypertensive conditions of pregnancy and coagulopathies.

- Have a low threshold for imaging modalities of investigation.

- Obtain an expert opinion timeously.

Given the SA situation, it is important for educational institutions to include training on headaches in general and particularly in pregnancy. The National Department of Health should provide guidelines on the management of headaches in pregnancy. It is surprising that, as headaches are such a common disorder, there are no guidelines on its management.

Acknowledgements. As these were case reports that illustrate lessons to learn, ethical clearance was not believed necessary. However, permission was sought from the National Department of Health to use the data. Author contributions. All authors contributed to the writing of this article and agreed to its submission.

Funding. None.

Conflicts of interest. None.

1. O'Neal MA. Headaches complicating pregnancy and the postpartum period. Pract Neurol 2017;17(3):191-202. https://doi.org/10.1136/practneurol-2016-001589

2. Robbins MS, Farmakidis C, Dayal AK, Lipton RB. Acute headaches diagnosis in pregnancy: A hospital study. Neurology 2015;85(12):1024-1030. https://doi.org/10.1212/WNL.0000000000001.954

3. Kane SC, Dennis A, da Silva Costa F, Kornman L, Brennecke S. Contemporary management of the cerebral complications of pre-eclampsia. Obstet Gynecol Int 2013;2013. https://doi.org/ $10.1155 / 2013 / 985606$

4. Knight M. Eclampsia in the United Kingdom 2005. Br J Obstet Gynaecol 2008;114(9):10721078

5. Fachinetti G, Alias R, DÁmico R, Beneditto C, Volpe A. The relationship between headache and preeclampsia: A case control study. Eur J Obstet Gynaecol Reprod Biol 2005;121(2):143-148. https://doi, org/10.1016/j.ejogrb.2004.12.020

6. Dangel AR, Atlas RO, Matsuo K. Headaches in pre-eclampsia: A clinical dilemma in diagnosing intracranial haemorrhage. Eur J Obstet Gynaecol Reprod Biol 2009;146(2):232-233. https://doi.org/ 10.1016/j.jogrb. 2009.06.006

7. Zeeman GG. Neurologic complications of pre-eclampsia. Semin Perinatol 2009;33(3):166-172. https:// doi.org/10.1053/j.semperi.2009.02.003

8. Patel SJ, Reede DL, Katz DS, et al. Imaging the pregnant patient for non-obstetric conditions: Algorithms and radiation dose considerations. Radiographics 2007;27(6):909-917. https://oi.org/ $10.1148 / \mathrm{rg} .276075002$

9. Wall BF, Hart D. Revised radiation doses for typical X-ray examinations. Br J Radiol 1997;70(833):437439. https://doi.org/10.1259/bjr.70.833.9227222.1148

10. McCollough CH, Scheuler BA, Arwell TD, et al. Radiation exposure and pregnancy: When should we be concerned? Radiographics 2007;27(4):909-917. https://doi.org/10.1148rg.274065149

11. Adeney KL, Williams MA, Miller RS, et al. Risk of pre-eclampsia in relation to maternal history of migraine headaches. J Matern Fetal Neonat Med 2005;18(3):167-172. https://doi.org/ $10.1080 / 14767050500260566$

12. Melhado EM, Maciel JA Jr, Guerreiro CA. Headaches during pregnancy. Can J Neurol Sci 2007;34(2):187-192. https://doi.org/0415227/0317-1671

Accepted 2 April 2018 